\title{
Atenção à puérpera com neurocisticercose e seu recém-nascido: relato de experiência
}

\section{Tatiane Fonseca Pereira ${ }^{1}$ \\ Mariana Viana Almeida ${ }^{2}$}

\footnotetext{
${ }^{1}$ Secretaria de Saúde do Distrito Federal. Residente do Programa de Residência Enfermagem em Obstetrícia

${ }^{2}$ Secretaria de Saúde do Distrito Federal. Tutora do Programa de Residência Enfermagem em Obstetrícia
}

Endereço para correspondência: Tatiane Fonseca Pereira. SGAN 906, bloco C, apartamento 214, Brasília, DF, Brasil. CEP: 70790-062. Telefone: (86) 99957-5531. Email: tfpereira2@gmail.com

\section{RESUMO}

Objetivo: relatar a experiência de uma residente em enfermagem obstétrica na assistência a uma puérpera com neurocisticercose e seu recém-nascido. Método: Atividade desenvolvida em um hospital público de Brasília em maio de 2020. Procedeu-se à Sistematização da Assistência de Enfermagem, embasada em discussão quanto aos achados e sua associação com evidências cientificas relativas à neurocisticercose. Resultados: A patologia em si não representou ameaça ao feto no caso abordado. A terapia medicamentosa mantida pela mãe durante a gestação implicou em riscos ao feto de malformações e sangramento, e riscos secundários na lactação. Conclusão: prestar assistência a casos incomuns como o retratado compreende um desafio o qual fomenta o desenvolvimento do raciocínio clínico e exercício da prática reflexiva.

Palavras-chave: Neurocisticercose; Gravidez; Anticonvulsivantes; Recém-nascido; Cuidados de enfermagem.

\section{Attention to the puerperal woman with neurocysticercosis and her newborn: experience report}

\begin{abstract}
Objective: to report the experience of a resident in obstetric nursing assisting a puerperal woman with neurocysticercosis and her newborn. Method: Activity developed in a public hospital in Brasilia in May 2020. The Nursing Care Systematization proceeded, based on a discussion about the findings and their association with scientific evidence related to neurocysticercosis. Results: the pathology itself did not pose a threat to the fetus in the case addressed. Drug therapy maintained by the mother during pregnancy represented risks to the fetus of malformations and bleeding, and secondary risks in lactation. Conclusion: providing
\end{abstract}


assistance to unusual cases like the one above represents a challenge which fosters the development of clinical reasoning and the exercise of reflective practice.

Keywords: Neurocysticercosis; Pregnancy; Anticonvulsants; Newborn; Nursing care

\section{INTRODUÇÃO}

A neurocisticercose (NCC) é uma helmintíase causada pelas larvas da Taenia Solium (TS), encontradas em carne suína contaminada ${ }^{1,2}$ Tal infecção do sistema nervoso central (SNC) constitui uma das etiologias mais comuns das convulsões em todo o mundo nos dias atuais $^{1,2}$. A TS é encontrada mundialmente em regiões onde porcos são criados, sem inspeção e controle sanitário, favorecendo o acesso destes às fezes humanas. Como área de prevalência elevada da neurocisticercose a America Latina é comumente apontada por vários autores, e dentre os países inclusos está o Brasil $2,3,4$.

O cestódeo causador tem como hospedeiros intermediários usuais os porcos, embora outras espécies possam acomodar as formas larvárias ${ }^{3,4}$ O ser humano, por outro lado, pode agir como hospedeiro intermediário ou definitivo. Dessa forma, a depender do ciclo de vida do cestódeo envolvido, duas infecções distintas podem ser desencadeadas no ser humano: teníase ou cisticercose $\mathrm{e}^{3,4}$. Na teníase, o ser humano atua como hospedeiro definitivo, e isso ocorre pela ingestão da forma larval (cisticercos) da TS, no consumo de carne suína contaminada, mal cozida. No intestino, os cisticercos amadurecem, transformando-se em tênias adultas, que se ligam à parede intestinal pela cabeça (escólex), de onde retiram seu alimento. À medida que crescem esses vermes produzem proglótides (segmentos) as quais são hermafroditas, e tornando-se prenhas se destacam da tênia repletos de ovos e são transmitidos ao ambiente através das fezes ${ }^{1,4}$.

Quando o homem ingere acidentalmente os ovos embrionários ou proglótides prenhas de TS, ele se torna hospedeiro intermediário e a infecção desenvolvida é a cisticercose $\mathrm{e}^{1,3}$. O contágio ocorre principalmente pela via fecal-oral por contato próximo com um portador de 
tênia ou por autocontaminação quando o homem também é portador da teníase. Os ovos ingeridos transformam-se em oncosferas, que penetram a parede intestinal, adentrando a corrente sanguínea e migram para os tecidos, evoluindo em seguida para cisticercos ${ }^{1,3}$.

Cisticercos podem ser identificados em qualquer parte do corpo, contudo são mais comumente detectados no cérebro, no fluido cérebro espinhal, musculatura esquelética, tecido subcutâneo ou no olho. A neurocisticercose é fruto do tropismo dessas larvas para o sistema nervoso central ${ }^{4}$.

A patologia deve ser considerada em mulheres grávidas ou no pós-parto com apresentações compatíveis, em especial, se provenientes de regiões endêmicas. O diagnóstico e manuseio da NCC durante a gravidez podem constituir um desafio, porém se intervenções rápidas forem tomadas, culminarão em possíveis melhores resultados maternos e fetais ${ }^{2}$. Tal manejo em gestantes é semelhante ao usualmente utilizado na população geral atingida, com a ressalva de que são necessárias considerações a respeito do bem-estar materno-fetal, em virtude dos desfechos que a patologia pode conceder ao binômio, quanto ao tratamento e possíveis manifestações clínicas com consequências para o feto ${ }^{2,5}$. Além disso, o puerpério e lactação também podem ser afetados 6 .

Considerando a prevalência da neurocisticercose no Brasil, suas singularidades no contexto materno-fetal e um número reduzido de pesquisas relacionadas à temática, este estudo se justifica pela necessidade de atualização direcionada ao manejo adequado e prestação de assistência holística pela enfermagem, com foco no desenvolvimento de habilidades e competências, e assim, proporcionar a sistematização da assistência de enfermagem baseada em evidências considerando as possíveis repercussões e achados materno-fetais decorrentes dessa patologia na gestação e puerpério.

Para nortear este estudo, traçou-se como objetivo, relatar a vivência de uma residente em enfermagem obstétrica no desenvolvimento de atividades inerentes à assistência a uma 
puérpera com neurocisticercose e seu recém-nascido durante atuação em um alojamento conjunto, associando achados na literatura à prática diária até a alta do binômio.

\section{MÉTODOS}

Trata-se de um relato de experiência a partir de atuação de enfermagem em rodízio de prática do programa de residência em Enfermagem em Obstetrícia. Este estudo apresenta a vivência de uma residente em enfermagem obstétrica do $1^{\circ}$ ano com desígnio de promoção de atenção prestada à puérpera e recém-nascido de forma holística, dando relevância à patologia materna e seus desenlaces ao binômio (mãe-bebê), durante internação hospitalar.

O desenvolvimento da atividade ocorreu do mês de maio a junho de 2020 na unidade alojamento conjunto de um hospital público do Distrito Federal (DF). Esse hospital é vinculado à Secretaria de Saúde do Distrito Federal (SES-DF) e compreende um dos campos de atuação do Programa de Residência Enfermagem em Obstetrícia. A ala de atuação se configura como de atendimento a puérperas e recém-nascidos e dispõe de 30 leitos. A assistência foi prestada durante os quatro dias em que ambos estiveram internados e durante este período se implementou a sistematização da assistência de enfermagem (SAE) com base nas taxonomias NANDA-NIC-NOC ${ }^{7,8}$.

O histórico de enfermagem foi levantado por meio de anamnese, exame físico e coleta de dados em prontuário eletrônico. E a partir deste, elencados os principais diagnósticos de enfermagem, bem como intervenções e resultados esperados. Evoluções de enfermagem foram registradas em prontuário eletrônico, as prescrições de enfermagem se basearam nas intervenções selecionadas e fora realizada avaliação do binômio no momento da alta. Alinhada

à SAE discutiu-se a associação dos achados com evidências cientificas relativas à neurocisticercose, na indagação das implicações materno-fetais durante gestação e puerpério para o caso relatado. 


\section{RESULTADOS E DISCUSSÃO}

Diante do exposto será apresentada a trajetória desde o contato inicial com o binômio até a alta, levantando reflexões pertinentes aos achados.

\section{ATENÇÃO À PUÉRPERA}

\section{Identificação}

L.G.S, sexo feminino, 33 anos, microempresária, procedente de São Sebastião (DF), tipo sanguíneo O positivo, G1P0A0, gestação por fertilização in vitro (FIV) visto que marido realizou vasectomia, pré-natal de alto risco no Hospital Materno infantil de Brasília - HMIB (11 consultas), por histórico patológico materno associado à gestação (Neurocisticercose e Hipotireoidismo) e anomalia fetal detectada por ecografia gestacional (cisto abdominal à direita medindo 2,3x2,3cm em US de 12/05/2020). A gestação é um fenômeno fisiológico e-sua evolução se dá na maior parte dos casos sem intercorrências. Quando a gestante é portadora de alguma doença, agravo ou os desenvolvem, com maiores chances de evolução desfavorável para o feto ou para a mãe, ela se enquadra no grupo de gestantes de alto risco ${ }^{9}$.

\section{HISTÓRICO PATOLÓGICO}

\section{Sintomatologia}

A neurocisticercose fora diagnosticada há aproximadamente 15 anos, com acompanhamento periódico pela neurologia desde então. A paciente citou cefaleia persistente, perda de equilíbrio e síncope como sintomas iniciais, progredindo para crises epiléticas sem perda de consciência. O acometimento pode provocar desde uma infecção completamente assintomática até doença grave e morte ${ }^{10}$. 
Na cisticercose, a apresentação clínica dependerá do número e localização dos cisticercos, bem como da extensão das respostas inflamatórias associadas, sendo as manifestações neuropsiquiátricas as mais comuns, entre elas, convulsões, distúrbio de comportamento e hipertensão intracraniana, esta última capaz de desencadear sintomas como cefaleia, náusea, vômitos, alterações da visão, tontura e confusão $0^{3,4}$.

\section{Diagnóstico}

A paciente estava sob posse de encaminhamento ao pré-natal de alto risco constando como descrição do neurologista: gestante portadora de neurocisticercose, última tomografia computadorizada (TC) de crânio (20/08/2018) evidenciando pequenas lesões calcificadas sem edema adjacente, compatíveis com neurocisticercose na forma nodular calcificada em região parietal esquerda, sem sintomas associados há 5 anos, em uso de fenobarbital $50 \mathrm{mg} / \mathrm{semana}-$ encaminho ao pré-natal de alto risco.

Os cisticercos são constituídos de duas partes principais, a parede vesicular e o escólex e estarão em estágio vesicular, ou seja, viáveis, ao entrarem no sistema nervoso central. Neste estágio os parasitas possuem uma membrana transparente, um líquido vesicular claro e um escólex invaginando estruturamente semelhante ao T. solium adulto. Estes cisticercos viáveis podem assim permanecer por anos ou iniciarem um processo de degeneração, como resultado da resposta imunológica do hospedeiro, que culmina em sua transformação em calcificações ${ }^{1,2}$. As convulsões se manifestam frequentes em pacientes com cistos degenerativos ${ }^{10}$.

A presença de calcificação não extingue a presença de inflamação ou a continuidade do mecanismo desencadeador da inflamação, edema e convulsões resultantes, causadas por cistos degenerativos, uma vez que estudos têm apontado que as calcificações não podem ser generalizadas como lesões inertes, sendo necessários monitoração e tratamento direcionado ${ }^{10}$. 
Neste caso a hipótese diagnóstica foi levantada a partir dos sintomas apresentados e confirmada com exame de imagem segundo relatos da paciente. O diagnóstico de NCC é clínico, epidemiológico e laboratorial. Logo, baseia-se na combinação de manifestações clínicas, estudos de imagem (raios X, TC e ressonância magnética), testes sorológicos e história de exposição $0^{3,4,11}$.

Os estudos sorológicos específicos no soro e líquido cefalorraquiano confirmam o diagnóstico da neurocisticercose. Enquanto os raios $\mathrm{X}$ identificam somente cisticercos em fase calcificada, a TC e ressonância magnética são capazes de identificar cisticercos nas diversas fases de seu desenvolvimento. Biópsia tecidual também possibilita a identificação microscópica da forma larvária ${ }^{3,12}$. Aspectos epidemiológicos incluem moradia ou viagens para áreas endêmicas, bem como contactuante próximo infectado por NCC. A cisticercose suína ainda que não esteja bem documentada é endêmica nas Américas, e no Brasil é cada vez mais diagnosticada, especialmente nas regiões Sul e Sudeste através dos serviços de neurologia, neurocirurgia e estudos anatomopatológicos. Em contrapartida, no Norte e Nordeste os casos em menor número podem ser justificados pela falta de notificação ${ }^{4}$.

\section{Tratamento}

Em relação ao tratamento, a paciente relatou uso de anti-helmíntico e anti-inflamatório associados após diagnóstico, os quais não soube informar os nomes, procedendo ao final deste com uso contínuo de anticonvulsivante. O manejo inicial da neurocisticercose deve ter como foco o tratamento sintomático das convulsões ou hidrocefalia, sendo que as convulsões geralmente são controladas com antiepiléticos ${ }^{3}$. Para o tratamento da helmintíase são utilizados antiparasitários, incluindo albendazol (15 mg/kg/dia, por 8-28 dias) ou praziquantel (50-100 $\mathrm{mg} / \mathrm{kg} / \mathrm{dia}$, em três doses fracionadas, por 15-30 dias). Uma combinação de albendazol e praziquantel $(50 \mathrm{mg} / \mathrm{kg} / \mathrm{dia})$ pode ser necessária em pacientes com lesões múltiplas ${ }^{4}$. 
Consequente à morte dos cisticercos ocorre uma resposta inflamatória em volta do parasita, sendo necessária associação de glicocorticoides como a dexametasona, ao tratamento, com intuito de reduzir tal resposta ${ }^{3,4}$.

Em pacientes com neurocisticercose parenquimatosa calcificada (NCP) a terapia sintomática isolada é recomendada em detrimento de antiparasitários ${ }^{12}$. A paciente em questão possuía cistos calcificados em região parietal esquerda, localizados, portanto em parênquima cerebral, enquadrando-se como NCP. Contudo, tratou a helmintíase logo após diagnóstico e não havia edema associado em neuroimagem recente que demandasse uso de corticoides, sendo o seguimento terapêutico com antiepilético associado à vigilância a conduta adequada para o caso.

A paciente possuía cistos calcificados em parênquima cerebral, porém sem descrição quantitativa que permitisse estimar um prognóstico. Isso porque o prognóstico da neurocisticercose depende da localização e a carga parasitária. Quando localizada em regiões subaracnoidea e intraventricular associam-se à mortalidade substancial e morbidade grave, enquanto o prognóstico da cisticercose cerebral parenquimatosa é afetado especialmente pela quantidade de lesões e extensão da inflamação ${ }^{10}$.

\section{GESTAÇÃO ATUAL}

Quando questionada, a paciente negou crises convulsivas ou episódios de síncope há mais de 5 anos, continuou fazendo uso do fenobarbital durante a gestação sob orientação médica, apresentou vertigem e cefaleia de fraca intensidade durante a gestação, não apresentando outros sintomas associados no decorrer do período gravídico.

Além da neurocisticercose, outra patologia referida nos antecedentes pessoais consistiu em hipotireoidismo pré-gestacional, com uso de levotiroxina $25 \mathrm{mcg} / \mathrm{dia}$, sem alterações percebidas ao longo da gestação por dosagem do hormônio tireoestimulante (TSH). Paciente 
negou alergias medicamentosas ou alimentares, bem como procedimentos cirúrgicos anteriores. Negou doenças crônicas na família. Negou etilismo, tabagismo ou uso de drogas. Durante a gestação fez uso de ácido fólico e sulfato ferroso, referiu infecção do trato urinário (bacteriúria assintomática) tratada com Ceftriaxona no $1^{\circ}$ trimestre com registros em caderneta de gestante. Registro de pressão arterial dentro dos valores de referências durante pré-natal. Sorologias: HIV e VDRL (não reagentes em $1^{\circ}$ e $3^{\circ}$ trimestres gestacionais), Hepatite B, Hepatite $\mathrm{C}$ e HTLV não reagentes, susceptível a toxoplasmose (IgG e $\operatorname{IgM}$ não reagentes em $1^{\circ}$ e $3^{\circ}$ trimestres gestacionais) e imune a citomegalovírus (IgG positivo e IgM negativo em $1^{\circ}$ trimestre).

Foi admitida às 7:30h do dia 24/05/2020 por amniorrexe prematura, em pródromos de trabalho de parto. Idade gestacional: 38 semanas e 5 dias por data da última menstruação (DUM). Testes rápidos HIV e VDRL não reagentes na admissão. Parto cesáreo ocorreu no mesmo dia, sem intercorrências, com indicação descrita no sistema: fertilização in vitro e acordo com a mãe pela equipe da obstetrícia de não indução. Início da cirurgia às 15 h55 e nascimento de RN vivo, do sexo feminino, às 16 h07 com índice de Apgar 9/9, peso ao nascer: 3125g, não necessária reanimação neonatal. Binômio encaminhado ao alojamento conjunto em tempo oportuno.

\section{AVALIAÇÃO DE ENFERMAGEM}

Puérpera acompanhada pela residente de enfermagem a partir do $1^{\circ}$ dia de pósoperatório que procede à avaliação de enfermagem: seguiu tranquila no leito, com seu recémnascido, acompanhada do esposo. Referia boa ingesta alimentar e hídrica, diurese e evacuações presentes e sem alterações. Deambulando com dificuldade por sensação dolorosa em sítio cirúrgico, contudo sem auxílio. Negou queixas álgicas no momento. Afirmava e demonstrava felicidade. Relatou que recém-nascido sugou seio materno, porém ela se queixou de dificuldade 
ao amamentar e pega dolorosa, relatou que o RN havia mamado há cerca de 2 horas. O vínculo do binômio se encontrava preservado.

Ao exame físico, a paciente se encontrava em bom estado geral, consciente, orientada, receptiva ao diálogo, pele hidratada, corada, mucosas oculares hipocoradas (+/4+). Normotensa (120x75mmHg), normocárdica (65 bpm), eupnéica (18 irpm), afebril $\left(36,3^{\circ} \mathrm{C}\right)$. Pescoço: linfonodos e massas não palpáveis. Ausculta pulmonar: murmúrio vesicular presente, sem ruídos adventícios. Ausculta cardíaca: ritmo regular em 2 tempos, bulhas normofonéticas, não auscultados sopros. Mamas simétricas, túrgidas, secretantes. Mamilos protrusos com presença de fissuras em estágio inicial. Abdome globoso, flácido, doloroso à palpação profunda. Globo de Pinard palpável em nível da cicatriz umbilical. Incisão cirúrgica limpa e seca, sem sinais flogísticos. Genitália íntegra, lóquios fisiológicos de coloração rubra, em quantidade característica. Eliminações vesicais presentes e intestinais ausentes até o momento. Extremidades: MMSS com boa perfusão tissular, ausência de edemas ou acessos venosos. MMII: ausência de edemas ou varizes, boa perfusão tissular periférica, preenchimento capilar $<$ 2s. Mobilidade e sensação tátil preservadas. Sinais de TVP ausentes (Sinal de Homans, Bandeira e Bancroft - negativos).

Ao final do exame físico foi avaliada a técnica de amamentação. Quando posicionado em seio materno, recém-nascido abocanhou apenas região mamilar, pega não sustentada e puérpera apresentava expressão facial de dor. A conduta de enfermagem foi interromper processo, ajustar pega e posicionamento para apreensão aréolo-mamilar, orientar puérpera quanto à sustentação da mama e prosseguir com demais orientações pertinentes à amamentação. O padrão de sucção evoluiu com melhora e paciente relatava diminuição de sensação dolorosa após ajustes. Após anamnese e exame físico, procederam-se com os demais passos do processo de enfermagem referentes à puérpera. 
Levando em consideração o histórico de enfermagem colhido (anamnese e exame físico) foram elencados os diagnósticos de enfermagem considerados mais relevantes, bem como as intervenções e resultados esperados (Tabela 1).

Tabela 1. Sistematização da assistência de enfermagem na atenção à puérpera, Brasília, DF, maio de 2020.

\begin{tabular}{lll}
\hline \hline Diagnósticos de Enfermagem & Intervenções de Enfermagem & Resultados Esperados
\end{tabular}

\begin{tabular}{|c|c|c|}
\hline $\begin{array}{l}\text { Amamentação ineficaz } \\
\text { relacionada à dor materna e } \\
\text { conhecimento insuficiente dos } \\
\text { pais sobre técnicas de } \\
\text { amamentação caracterizada } \\
\text { por resistência do lactente em } \\
\text { apreender a região areolar- } \\
\text { mamilar; sucção na mama } \\
\text { não sustentada; persistência } \\
\text { de mamilos doloridos; e } \\
\text { esvaziamento insuficiente de } \\
\text { cada mama por } \\
\text { amamentação }\end{array}$ & $\begin{array}{ll}\text { - } & \text { Assistência na amamentação } \\
\text { - } & \text { Controle da dor } \\
\text { - } & \text { Orientações aos pais } \\
\text { - } & \text { Aconselhamento para lactação } \\
\text { - } & \text { Promoção do envolvimento familiar } \\
\text { - } & \text { Cuidados da pele: tratamento tópico } \\
\text { - } & \text { Supervisão da pele } \\
\text { - } & \text { Orientada a procurar o Banco de leite após } \\
& \text { a alta }\end{array}$ & $\begin{array}{l}\text { Conhecimento: } \\
\text { amamentação } \\
\text { (alcance } \\
\text { compreensão } \\
\text { transmitida sobre a } \\
\text { lactação e a nutrição } \\
\text { do bebê por meio da } \\
\text { amamentação) }\end{array}$ \\
\hline 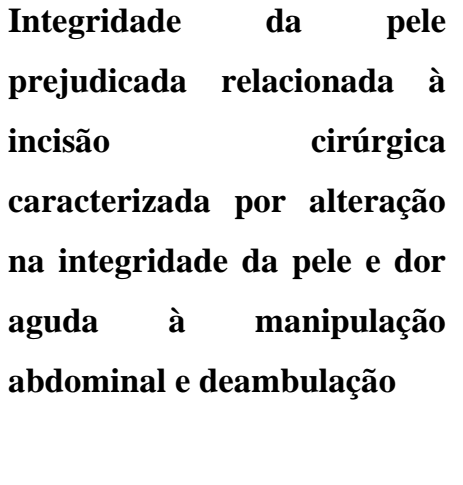 & $\begin{array}{l}\text { - Cuidados com local de incisão } \\
\text { (higienização, monitoramento e promoção } \\
\text { da cicatrização da ferida fechada por } \\
\text { suturas, clipes ou grampos) } \\
\text { - } \quad \text { Supervisão da pele } \\
\text { - } \text { Cuidados no pós-parto cesáreo } \\
\text { - } \text { Proteção contra infecção } \\
\text { - } \quad \text { Administração de medicamentos }\end{array}$ & $\begin{array}{l}\text { - } \text { Cicatrização de } \\
\text { feridas: primeira } \\
\text { intenção (alcance da } \\
\text { regeneração de } \\
\text { células e tecidos após } \\
\text { fechamento } \\
\text { intencional) }\end{array}$ \\
\hline $\begin{array}{l}\text { Deambulação prejudicada } \\
\text { relacionada à dor } \\
\text { caracterizada por capacidade } \\
\text { prejudicada de andar uma } \\
\text { distância necessária }\end{array}$ & $\begin{array}{ll}\text { - } & \text { Terapia com exercício: deambulação } \\
\text { - } & \text { Administração de analgésicos } \\
\text { - } & \text { Controle do ambiente: segurança }\end{array}$ & $\begin{array}{l}\text { Mobilidade } \\
\text { (capacidade de } \\
\text { movimentar-se pelo } \\
\text { próprio ambiente, de } \\
\text { forma independente, }\end{array}$ \\
\hline
\end{tabular}




\begin{tabular}{|c|c|c|}
\hline & & $\begin{array}{l}\text { com ou sem } \\
\text { dispositivo auxiliar) }\end{array}$ \\
\hline $\begin{array}{l}\text { Risco de queda relacionado a } \\
\text { dificuldades na marcha, } \\
\text { história de quedas e alteração } \\
\text { neurológica } \\
\text { (neurocisticercose) }\end{array}$ & 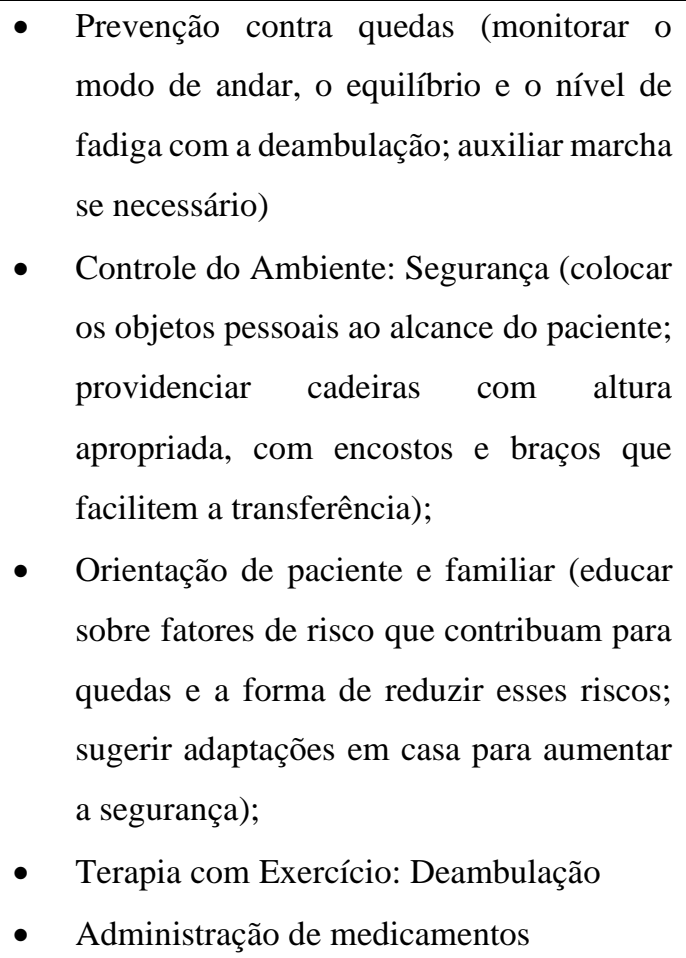 & $\begin{array}{l}\text { - } \text { Comportamento de } \\
\text { Prevenção de Quedas }\end{array}$ \\
\hline $\begin{array}{l}\text { Risco de infecção relacionado } \\
\text { a procedimento invasivo e } \\
\text { integridade da pele } \\
\text { prejudicada }\end{array}$ & $\begin{array}{ll}\text { - } & \text { Cuidados Pós-Parto } \\
\text { - } & \text { Cuidados da Pele: Local de incisão }\end{array}$ & $\begin{array}{l}\text { - Controle de Riscos: } \\
\text { Processo Infeccioso } \\
\text { - Cicatrização de } \\
\text { Feridas: Primeira } \\
\text { Intenção }\end{array}$ \\
\hline
\end{tabular}

FONTE: Pesquisa direta

\section{ATENÇÃO AO RECÉM-NASCIDO}

Após avaliação da puérpera, procedeu-se à do recém-nascido. Durante avaliação estava em alojamento conjunto com a mãe, em lactação materna exclusiva (LME). Diurese e evacuações presentes e sem alterações no turno, segundo relatos da mãe. Ao exame físico não apresentou alterações, sinais vitais dentro do padrão de normalidade. Como alteração pertinente ao $\mathrm{RN}$ havia a amamentação prejudicada, citada anteriormente na atenção voltada à puérpera. O histórico foi complementado com informações coletadas em prontuário eletrônico. Tipo sanguíneo: $\mathrm{AB}+$. 
Como conduta médica inicial fora solicitada rotina de ALCON, solicitada USG de abdome total para elucidação do cisto abdominal relatado em ecografia gestacional e prescrição de dose secundária de vitamina $\mathrm{K}$ devido ao uso materno de fenobarbital. Algumas drogas anticonvulsivantes, dentre elas, o fenobarbital, agem diminuindo o transporte da vitamina $\mathrm{K}$, que possui ação anticoagulante, através da placenta, podendo ocasionar risco de hemorragia fetal e neonatal aumentados. Nesses casos, é recomendação administrar à mãe $20 \mathrm{mg} / \mathrm{dia}$ de vitamina $\mathrm{K}$ durante o último mês de gravidez e $1 \mathrm{mg}$ IM ao recém-nascido9 .

Procedeu-se com os passos seguintes da SAE após levantamento do histórico do RN (Tabela 2).

Tabela 2. Sistematização da Assistência de Enfermagem na atenção ao recém-nascido, Brasília, DF, maio de 2020.

\begin{tabular}{lclc}
\hline \hline Diagnósticos de Enfermagem & Intervenções de Enfermagem & Resultados Esperados \\
\hline \hline Padrão ineficaz de & $\bullet$ & Assistência na amamentação & Manutenção da \\
alimentação do lactente & $\bullet$ & Orientações aos pais & amamentação \\
relacionado à amamentação & $\bullet$ & Monitoração nutricional & \\
ineficaz caracterizado por & $\bullet$ & Controle do peso & \\
capacidade prejudicada de & & & \\
manter uma sucção eficaz & & Precauções contra sangramento & Respostas à medicação \\
\hline Risco de sangramento & $\bullet$ & Controle de medicamentos & Controles de risco \\
relacionado à complicação & $\bullet$ & Identificação de riscos (realização de & \\
gestacional & & procedimentos, avaliação de exames & \\
\hline & & laboratoriais) & \\
\hline
\end{tabular}




\begin{tabular}{lllll}
\hline Risco de hiperbilirrubinemia & $\bullet$ & Monitoramento do recém-nascido & $\bullet$ & Adaptação do Recém- \\
neonatal relacionado à & & (exame físico, exames laboratoriais, & Nascido \\
anomalia em lactente, & padrão de diurese e evacuação) & Risco de icterícia neonatal \\
lactente com nutrição & $\bullet$ & Assistência na amamentação & diminuído \\
inadequada e & & & \\
incompatibilidade de tipo & & & \\
sanguíneo entre a mãe e o & & & \\
lactente & $\bullet$ & Controle do Ambiente: Segurança & Comportamento de \\
\hline Risco de queda em neonato & $\bullet$ & Manutenção terapia medicamentosa & \\
relacionado à condição & & materna & \\
patológica materna & & & \\
\hline \hline
\end{tabular}

FONTE: Pesquisa direta

\section{REPERCUSSÕES E DESFECHO MATERNO-FETAIS}

Há poucos relatos de casos de neurocisticercose na gravidez, mas sabe-se que o manejo dependerá da clínica manifestada pela paciente, com enfoque em terapia sintomática. Seu diagnóstico durante a gestação e o pós-parto é difícil e advém de um alto nível de suspeita, e na presença de convulsões entre segundo semestre gestacional e puerpério. É necessário diagnóstico diferencial de eclampsia visto a sintomatologia similar, mediante avaliação completa, exames de neuroimagem e análise de fatores epidemiológicos de risco ${ }^{2,12,13,14}$. No caso da infecção materna por TS, a transmissão vertical durante a gestação, o trabalho de parto, parto ou amamentação não é sustentada por evidências, contudo é possível que haja transmissão horizontal do agente etiológico seja por meio de contato direto, contato com secreções ou fômites contaminados, tornando dessa forma crucial a triagem de mãe com NCC para a teníase ${ }^{13}$. 
Gestações em mulheres com neurocisticercose, em sua maioria, estão associadas a bons desfechos e não há evidências de que a própria patologia desencadeie malformações fetais ou danos neurológicos. A epilepsia também não é considerada prejudicial para a gravidez, excetuando-se os casos de convulsões prolongadas as quais representam um risco aumentado de hipóxia ao feto. Possíveis repercussões negativas são atribuídas aos efeitos dos medicamentos antiepiléticos, por sua associação a malformações fetais ${ }^{9,13}$.

Os medicamentos antiepiléticos (DEAs) são iniciados ou têm continuidade terapêutica no período gravídico com intuito de evitar crises recorrentes. Contudo, pelo fato de cruzarem a barreira placentária e existirem evidências dos efeitos teratogênicos, o uso de tais medicamentos representa um risco para o desenvolvimento físico e neurológico do feto. Dessa forma, a decisão pelo tratamento na gestação requer avaliação risco-benefício ${ }^{5,13,15}$. É preferível optar pela monoterapia, uma vez que as combinações de antiepiléticos, em especial com ácido valpróico, associam-se a maior incidência de malformações se utilizadas no primeiro trimestre. O risco aumentado de defeitos congênitos está relacionado a altas dosagens e a politerapia, e apenas uma pequena parcela de pacientes com NCC não desenvolverá adequado controle das crises com monoterapia ${ }^{11,13,15}$.

Categoria de risco na gravidez compreende um enquadramento desenvolvido pela Food and Drug Administration (FDA) em que os fármacos são categorizados a partir do risco de causar dano ao feto durante a gravidez, baseando-se em estudos em animais ou humanos. Dentre as cinco categorias descritas (A, B, C, D e X), o fenobarbital é alocado na categoria D de risco na gravidez, significando que o fármaco demonstrou evidências positivas de risco fetal humano, porém, os benefícios potenciais para a mulher, eventualmente, justificam o risco, como em casos de doenças graves ou que ameaçam a vida, ou quando não existam outras drogas mais seguras ${ }^{16}$. Ademais, estudos apontam que dentre as malformações comumente associadas ao 
uso do fenobarbital podem ser apontadas malformações cardíacas, retardo de crescimento e presença de fenda labial ${ }^{15}$.

A terapia antiparasitária pode estar associada ao agravamento precoce da atividade convulsiva por precipitar ou aumentar a inflamação e o edema cerebral, sendo preferível adiar para o pós-parto. Benefícios e riscos da terapia antiparasitária durante a gestação devem ser discutidos com a paciente, porém se a condição clínica for grave o suficiente tal que justifique seu uso, o tratamento de escolha é com praziquantel em detrimento ao albendazol, uma vez que este último apresenta um perfil teratogênico maior ${ }^{2,13}$. Os corticosteroides podem ser usados na gravidez, se necessários, e devem ser associados à terapia antiparasitária para controle do edema secundário à resposta imune aos parasitas em degeneração, contudo devem ser evitados em pacientes com calcificações devido à piora dos sintomas após a diminuição gradual da medicação ${ }^{13,14}$.

Quanto à lactação, pequenas quantidades de praziquantel são excretadas no leite materno, portanto recomenda-se aguardar 72 horas após o uso antes da retomada à amamentação ${ }^{6}$.

Em decorrência da limitação de estudos sobre a segurança dos DEAs durante a lactação, as mães com epilepsia recebem conselhos conflitantes sobre amamentação com frequência, sendo desafiador a escolha entre uma fonte artificial de alimento ou a exposição do recémnascido a um fármaco capaz de atuar no sistema nervoso ${ }^{6}$. A literatura aponta que o lactente é afetado, se qualquer medicamento usado pela mãe for capaz de ser transferido pelo leite materno, atingindo a circulação sanguínea sistêmica da criança em uma quantidade suficiente para produzir um efeito farmacológico. Tais drogas que são excretadas no leite materno em quantidades significativas se caracterizam por altas concentrações plasmáticas maternas, baixo grau de ligação às proteínas, baixo peso molecular e alta solubilidade em gordura e, dentre os 
medicamentos com potencial de alcançar níveis séricos significativos em lactentes está o fenobarbital ${ }^{6}$.

Fenobarbital, antiepilético utilizado pela paciente do caso relatado, é contraindicado durante o aleitamento, pois é excretado no leite humano podendo gerar reações adversas à criança. Logo, o profissional médico deve prescrever alternativas para o seu tratamento ou para a alimentação do bebê, e na inexistência de alternativa de tratamento, o aleitamento materno deverá ser interrompido ${ }^{16}$. Ele possui transferência baixa a moderada para o leite materno. Em virtude de uma meia-vida extremamente longa e o baixo grau de ligação às proteínas em neonatos, o fenobarbital tem potencial acumulativo durante a amamentação. No entanto, a dose teórica estimada para produzir efeito farmacológico é muito menor que a dosagem pediátrica recomendada. Dessa forma, o tratamento com fenobarbital pode ser considerado compatível com a amamentação, desde haja monitoramento cuidadoso dos lactentes e em caso de suspeita de efeitos colaterais, como sonolência ou baixo ganho de peso, opta-se limitar ou interromper a amamentação ${ }^{6}$.

Até o momento da alta, o RN em questão não apresentou malformações aparentes. Ecografia evidenciou cisto de colédoco, que apesar de consistir em uma anomalia, não têm evidências científicas de sua associação ao uso do fenobarbital pela mãe na gestação. Os exames laboratoriais do recém-nascido solicitados apresentaram resultado dentro dos padrões de normalidade. Após os achados, a equipe médica decidiu por alta hospitalar com encaminhamento para seguimento ambulatorial com a equipe de cirurgia pediátrica do mesmo hospital.

Recomendações de amamentação exclusiva e manutenção de uso de fenobarbital materno, justificado pelo bem estar do RN na alta e o uso regular da medicação pela puérpera, porém com monitoramento cuidadoso posterior à alta, solução citada na literatura. O padrão de amamentação se apresentou adequado, puérpera sem queixas no momento da alta, contato do 
banco de leite da sua referência, repassado pelo serviço para acompanhamento posterior se necessário, binômio sem motivos para manutenção da internação e com encaminhamento para acompanhamento ambulatorial

em

mãos.

\section{CONCLUSÕES}

A atuação em alojamento conjunto se constitui em uma estratégia que oportuniza o aprendizado do residente em enfermagem obstétrica mediante prestação de assistência a casos diversificados, fomentando o desenvolvimento do raciocínio clínico direcionado não unicamente a um paciente, mas ao binômio mãe-bebê. Alia-se a isso, a oportuna implementação da sistematização da assistência de enfermagem e o uso da criatividade na resolução dos problemas. Alinhar teoria a uma situação real conduz à proposição de intervenções fundamentadas e ao exercício da prática reflexiva. Ademais, acompanhar um caso atípico como o relatado constitui um desafio ao provocar fuga à rotina de atuação, mas também conduz ao aprimoramento profissional ao instigar busca aprofundada do conhecimento e promoção de assistência holística que alia teoria e prática.

\section{REFERÊNCIAS}

1 Del Brutto OH. Neurocysticercosis. Neurohospitalist. 2014; 4(4):205-212. Disponível em: https://doi.org/10.1177/1941874414533351

2 D'Cruz RF, Ng SM, Dassan P. Neurocysticercosis in pregnancy: maternal and fetal outcomes. Oxf Med Case Reports. 2016; 2016(7):138-140. Disponível em: https://doi.org/10.1093/omcr/omw019

3 Kasper DL, Fauci AS, Hauser SL, Longo DL, Jameson JL, Loscalzo J. Manual de Medicina de Harrison [recurso eletrônico] 19. ed. Porto Alegre:AMGH, 2017. 
4 Brasil. Ministério da Saúde. Secretaria de Vigilância em Saúde. Departamento de Vigilância Epidemiológica. Doenças infecciosas e parasitárias: guia de bolso. 8. ed. rev. Brasília: Ministério da Saúde, 2010. 444 p

5 Weston J, Bromley R, Jackson CF, Adab N, Clayton-Smith J, Greenhalgh J, et al. Monotherapy treatment of epilepsy in pregnancy: congenital malformation outcomes in the child. Cochrane Database Syst Rev. 2016;11(11):CD010224. https://doi.org/10.1002/14651858.CD010224.pub2

6 Veiby, G, Bjørk, M, Engelsen, BA, Gilhus, NE. Epilepsy and recommendations for breastfeeding. $\quad$ Seizure. 2015; 28:57-65. Disponível em: https://doi.org/10.1016/j.seizure.2015.02.013

7 North American Nursing Diagnosis Association International. Diagnósticos de enfermagem da NANDA: definições e classificação 2018 - 2020. [recurso eletrônico] 11.ed. Porto Alegre: Artmed; 2018. Disponível em: http://nascecme.com.br/2014/wpcontent/uploads/2018/08/NANDA-I-2018_2020.pdf. Acesso em junho de 2020.

8 Jonhson M, Moorhead S, Bulechek G, Butcher H, Maas M, Swanson E. Ligações NANDA-NOC-NIC: condições clínicas, suporte ao raciocínio e assistência de qualidade. Rio de Janeiro: Elsevier, 2012.

9 Brasil. Ministério da Saúde. Secretaria de Atenção à Saúde. Departamento de Ações Programáticas Estratégicas. Gestação de alto risco: manual técnico. 5. ed. Brasília: Editora do Ministério da Saúde, 2012. 302 p.

10 Garcia HH, Nash TE, Del Brutto OH. Clinical symptoms, diagnosis, and treatment of neurocysticercosis. Lancet Neurol. 2014;13(12):1202-1215. Disponível em: https://doi.org/10.1016/S1474-4422(14)70094-8

11 Rajshekhar V. Neurocysticercosis: Diagnostic problems \& current therapeutic strategies. Indian J Med Res. 2016;144(3):319-326. DOI: 10.4103/0971-5916.198686 
12 White AC Jr, Coyle CM, Rajshekhar V, Singh G, Hauser WA, Mohanty A, et al. Diagnosis and Treatment of Neurocysticercosis: 2017 Clinical Practice Guidelines by the Infectious Diseases Society of America (IDSA) and the American Society of Tropical Medicine and Hygiene (ASTMH). Clin Infect Dis. 2018;66(8):e49-e75. Disponível: https://doi.org/10.1093/cid/cix1084

13 Webb C, Rosa M, Olson G, Cabada M. Neurocysticercosis in Pregnancy. AJP Rep. 2018;8(2):e51-e56. doi:10.1055/s-0038-1639615

14 Hall RL, Anderson B, Schulkin J, Cantey PT, Montgomery SP, Jones JL. Survey of Obstetrician-Gynecologists in the United States About Taeniasis and Cysticercosis. Am J Trop Med Hyg. 2017;96(1):233-242. DOI: https://doi.org/10.4269/ajtmh.16-0350

15 Veroniki AA, Cogo E, Rios P, Straus SE, Finkelstein Y, Kealey R, et al. Comparative safety of anti-epileptic drugs during pregnancy: a systematic review and network meta-analysis of congenital malformations and prenatal outcomes. BMC Med. 2017;15(1):95. https://doi.org/10.1186/s12916-017-0845-1

16 Agência Nacional de Vigilância Sanitária (Brasil). $R D C N^{\circ} 60$, de 17 de dezembro de 2010. Estabelece frases de alerta para princípios ativos e excipientes em bulas e rotulagem de medicamentos. Diário Oficial da União 16 dez 2010 [Acesso em 03 jul 2020]. Disponível em: http://bvsms.saude.gov.br/bvs/saudelegis/anvisa/2010/res0060_17_12_2010.html 